\title{
Uso de aprendizado de máquina para criação de um Banco de Dados com informações taxonômicas de gene de rRNA $16 \mathrm{~s}$
}

\author{
Pâmela M. Rezende* Joicymara S. Xavier** \\ Adriano Alonso Veloso ${ }^{* * *}$ Gabriel R. Fernandes ${ }^{* * * *}$ \\ Douglas Eduardo V. Pires ${ }^{* * * *}$ \\ * Instituto de Ciências Biológicas, Universidade Federal de Minas \\ Gerais, MG, (e-mail: pamela.m.rezende@gmail.com) \\ ** Instituto de Ciências Biológicas, Universidade Federal de Minas \\ Gerais, MG, (e-mail: joicy.xavier7@gmail.com) \\ *** Departamento de Ciência da Computação, Universidade Federal de \\ Minas Gerais, MG, (e-mail: adrianov@dcc.ufmg.br) \\ **** Instituto René Rachou, FIOCRUZ Minas, MG
}

\begin{abstract}
Next generation sequencing techniques produce a large volume of biological data. It is impossible to process these data manually and sometimes it is still processed with errors and mismatches. Thus, to minimize such problems, this project aims to create a curated and reliable database of $16 \mathrm{~s}$ rRNA sequences for taxonomic classification using computational techniques such as machine learning.

Resumo: Técnicas de sequenciamento de nova geração produzem um grande volume de dados biológicos. É impossível processar esses dados manualmente e algumas vezes, eles ainda são processados com erros e discordâncias. Assim, para minimizar tais problemas, este projeto tem como objetivo criar uma base de dados curada e confiável de sequências do rRNA 16s para classificação taxonômica, utilizando técnicas computacionais como o aprendizado de máquina.
\end{abstract}

Keywords: Machine learning, semi-supervised learning, unbalance, hierarchical classification, biological data.

Palavras-chaves: Aprendizado de máquina, aprendizado semi-supervisionado, desbalanceamento, classificação hierárquica, dados biológicos.

\section{INTRODUÇÃO}

Populações microbianas são altamente diversificadas e membros-chave de muitos ecossistemas de importância global. Entretanto, métodos de cultura clássicos permitem a caracterização de menos de 0,1-1\% da diversidade microbiana em grandes ecossistemas (Ravin et al., 2015). Através das técnicas de sequenciamento do DNA é possível focar no estudo de populações microbianas não cultiváveis, o que favoreceu o surgimento da metagenômica (Handelsman, 2004).

Estima-se que a população microbiana celular do genoma humano ultrapassa o número de células humanas em 10 vezes (Kurokawa et al., 2007). Desde a concepção de técnicas de sequenciamento em larga escala, o uso da área de metagenômica tem aumentado significativamente, gerando assim um grande volume de dados e o aumento da necessidade de utilização das técnicas computacionais para análise das mesmas (Bálint et al., 2016).

Técnicas computacionais para metagenômica envolvem considerações sobre: montagem, predição de genes, assina-

\footnotetext{
* Nossos sinceros agradecimentos à CAPES e FAPEMIG pelo suporte financeiro a essa pesquisa.
}

tura taxonômica, estimativa de biodiversidade, previsões de funções, integração de dados e várias outras (Teeling and Glöckner, 2012). Um dos desafios básicos enfrentados pela área é a classificação taxonômica das sequências (Sedlar et al., 2017), dado que, devido ao sequenciamento em larga escala, o volume de dados provenientes do processo é grande.

A taxonomia comumente classifica os organismos em sete níveis: domínio, filo, classe, ordem, família, gênero e espécie (Thompson et al., 2013). Essa classificação baseia-se em sequências de genes marcadores que apresentam uma presença universal e conservada. Em organismos procariotos o gene marcado mais comumente utilizado é o rRNA $16 \mathrm{~s}$ (Nguyen et al., 2016). A assinatura taxonômica é gerada a partir de bases de dados com sequências de 16 s já classificadas. Existem várias bases de dados que disponibilizam classificações taxonômicas baseada em diversas técnicas de agrupamentos de sequências. As mais utilizadas para assinatura de procariotos são: GreenGenes (DeS, 2006), NCBI (National Center for Biotechnology Information) (Coordinators, 2013), RDP (Ribosomal Database Project) (col, 2014) e SILVA (Yilmaz et al., 2014).

A classificação taxonômica do Greengenes é dedicada à Bacteria e Archaea. Ele é baseado na construção automá- 
tica de novas árvores filogenéticas e no mapeamento de assinatura taxonômica de outras bases, como por exemplo o NCBI. A sua última atualização foi em maio de 2013. O NCBI não é apenas um banco de taxonomia, mas contém nomes de todos os organismos associados às sequências. Ele é atualizado através de curadoria manual da literatura atual e usa mais de 150 fontes. Os arquivos de classificação taxonômica do NCBI são atualizados diariamente.

A base de dados RDP é baseada em seqüências 16S rRNA de Bacteria, Archaea e Fungi (Eukarya) e possui sequências de $16 \mathrm{~S}$ disponíveis nas bases de dados do INSDC (International Nucleotide Sequence Database Collaboration) (Nakamura et al., 2013). As informações sobre a classificação taxonômica de procariotos são baseadas no Manual de Bergey (Evtushenko et al., 2015) e na Lista de Nomes Prokarióticos com Nomenclatura Permanente (LPSN) (Parte, 2014). A última atualização da base foi divulgada em setembro de 2016 (RDP 11).

A base de dados SILVA também contém informações taxonômicas para os domínios de Bacteria, Archaea e Eukarya. Assim como o RDP, o SILVA utiliza para classificação dos procariotos o Manual de Bergey (Evtushenko et al., 2015) e a LPSN (Parte, 2014), além disso, as atribuições de classificação taxonômica são realizadas manualmente (Yilmaz et al., 2014). A última versão da atualização do SILVA, o SILVA 132, se deu em julho de 2017.

As bases de dados citadas possuem discordâncias entre si em todos os níveis taxonômicos (Balvočiūtè and Huson, 2017) além de apresentarem divergência também em suas classificações taxonômicas para uma mesma sequência. Algumas bases apresentam nomes duplicados, representando assim, diferentes organismos (Coordinators, 2013). Em sua maioria, essas bases não possuem uma curadoria manual das assinaturas taxônomicas de todas as sequências, causando assim vieses em projetos metagenômicos de microrganimos que as utilizam.

\section{TRABALHOS RELACIONADOS}

A taxonomia e classificação têm alta importância para a ecologia microbiana, porém, todas as abordagens existentes possuem limitações (Beiko, 2015). O gene 16s é facilmente avaliado em massa, mas está sujeito a erros devido ao processo de amplificação e resolução limitada. Sinais conflitantes podem produzir resultados indesejáveis e, nos casos onde a sequência do genoma e outros atributos moleculares entram em divergência devem haver critérios claros para relatar e lidar com esses conflitos (Beiko, 2015).

É notório que existem muitos desafios para a taxonomia. Nesse sentido, algumas abordagens tem sido desenvolvidas com o objetivo de contornar, prever ou corrigir erros oriundos de sequenciamento. Alguns exemplos são: QIIME (Kuczynski et al., 2011), DADA2 (Callahan et al., 2016) e TAG.ME (Pires et al., 2018).

QIIME, acrônimo para Quantitative Insights Into Microbial Ecology é uma aplicação de software que realiza análises da comunidade microbiana. A ferramenta tem sido utilizada para analisar e interpretar dados de sequência de ácidos nucleicos de fungos, vírus, bactérias e arqueias. Já o DADA2 (Divisive Amplicon Denoising Algorithm foca na modelagem e correção dos erros de amplificação de sequências Illumina. O DADA2 infere sequências da amostra e resolve diferenças de até 1 nucleotídeo, diminuindo consideravelmente a quantidade de sequências espúrias.

Trabalhos mais recentes têm utilizado modelos preditivos para tratar problemas específicos. Como é o caso do TAG.ME (Taxonomic Assignment of Genetic Markers for Ecology). TAG.ME é um método inovador independente de alinhamento e de amplificação específica para tarefas taxonômicas baseadas em marcadores genéticos. Ele usa uma abordagem de aprendizado supervisionado multicamadas para atribuir taxonomia baseado em genes de marcadores genéticos definidos pelo usuário.

O TAG.ME se mostrou um método eficiente para atribuição de taxonomia. Porém, principalmente a nível de espécies, Pires et al. (2018) mostra que ainda existem muitas sequências que não conseguem ser classificadas por ele e pelas principais metodologias existentes. Como essas sequências não estão rotuladas nas bases de dados, ou estão rotuladas de forma errada, uma abordagem que conseguisse corrigi-las poderia aumentar ainda mais a eficiência dos métodos que os utilizam. Sendo assim, métodos de aprendizado de máquina ativos poderiam ser uma opção.

Aprendizado de máquina é uma ciência que utiliza métodos computacionais e estatística para analisar dados. As técnicas de aprendizado de máquina surgiram como um meio que pudesse permitir que os computadores aprendessem sem necessariamente ter sido programado instrução por instrução. A ideia básica por trás do aprendizado de máquina é fornecer dados para que o computador possa aprender com eles.

Dentre as abordagens do aprendizado de máquina, destacase o de aprendizado de máquina supervisionado, que foca em problemas de previsão: tendo uma base de dados com "alvos" para cada observação (pares $x, y$ ), a meta é aprender quais "alvos" $(y)$ estão associados a quais dados $(x)$. Isso é feito apresentando ao computador pares suficientes de dados e alvos, até que ele aprenda a associar um ao outro.

Uma limitação do aprendizado de máquina supervisionado é que pode ser extremamente custoso conseguir os alvos para cada observação, ou eles estarem errados, ou a quantidade ser insuficiente. Uma opção nesse caso é utilizar o aprendizado de máquina semi-supervisionado, em que considera-se apenas uma pequena parcela dos dados como estando rotulados. O aprendizado ativo, que é um método de aprendizagem semi-supervisionada, é recomendado quando se tem dados rotulados mas é necessário diminuir o tempo de computação e quando se tem classes desequilibradas na variável de destino (Settles, 2009).

No aprendizado ativo, o modelo escolhe os dados de treino dos quais aprenderá mais, levando em consideração que o modelo irá prever melhor o conjunto de testes com menos dados se for incentivado a escolher as amostras com as quais deseja aprender. (Settles, 2009). Em geral, um modelo de aprendizado ativo primeiro é treinado por um classificador (regressão logística, floresta aleatória, SVM, etc) em uma seleção de amostras aleatórias rotuladas manualmente. Depois, os rótulos para o restante dos dados são previstos utilizando este modelo. A seguir, o modelo é 
treinado em uma série de loops, seguindo o seguinte fluxo: Loop 1:

- Identificar as amostras em que o modelo estava mais incerto;

- Pegar as amostras rotuladas por um humano;

- Treinar novamente o classificador usando as amostras aleatórias iniciais mais as amostras do rotuladas por humano;

- Prever novos rótulos para o restante dos dados utilizando o modelo melhorado.

Loop 2: Repetir o processo do Loop 1.

Na prática, saber quando parar o loop é uma tarefa difícil uma vez que não há uma estrutura teórica documentada que informe o número de loops necessários. Essa escolha irá depender da métrica utilizada para avaliação e também da aplicação.

Diante do exposto, em estudos metagenômicos, a utilização de uma base de dados curada e confiável auxilia na confirmação da estrutura taxonômica do organismo de interesse. Neste sentido, esse projeto tem como objetivo, criar uma base de dados curada e confiável de sequências do gene codificador do rRNA 16s para classificação taxonômica, utilizando técnicas de aprendizado de máquina.

\section{METODOLOGIA}

A metodologia utilizada para este trabalho é mostrada na Figura 1. Inicialmente foi feita a avaliação da base que irá gerar a base de dados curada ao final do projeto. Depois, foram geradas as características de cada sequência, baseado no cálculo de frequência de tetra nucleotídeos. Após a geração das características, as sequências foram pré-processadas afim de retirar dados com classificação errada ou não rotulados. Através de uma análise exploratória dos dados foi avaliada a classificação de cada rotulagem. Em pararelo, os dados rotulados corretamente foram submetidos a classificadores e avaliados. As outras etapas mostradas no fluxograma ainda serão executadas. Cada uma das etapas é descrita em detalhes nas subseções a seguir.

\subsection{Avaliação da base de dados}

A base de dados escolhida para realizar a curadoria proposta foi a SILVA. Os dados desta base são representados por sequências biológicas compostas por um conjunto de nucleotídeos (A, C, U e G) que identifica cada organismo. Um exemplo de sequência pode ser visto pela Figura 4. O rótulo para cada organismo é dado pela assinatura taxonômica seguindo o padrão: $k$ _Reino; $p_{\text {_Filo; }}$ __Classe; o_Ordem; f_Familia; g_Gênero; s_Espécie

Como a rotulagem é hierárquica, ou seja, a classificação de um nível depende do nível inferior, foi selecionado, a princípio, apenas dados a nível de espécie.Sequências que não estão classificadas a nível de espécie serão reclassificadas futuramente.

\subsection{Geração das características}

As características para agrupamento taxonômico são baseados em duas estratégias: dependente e independente

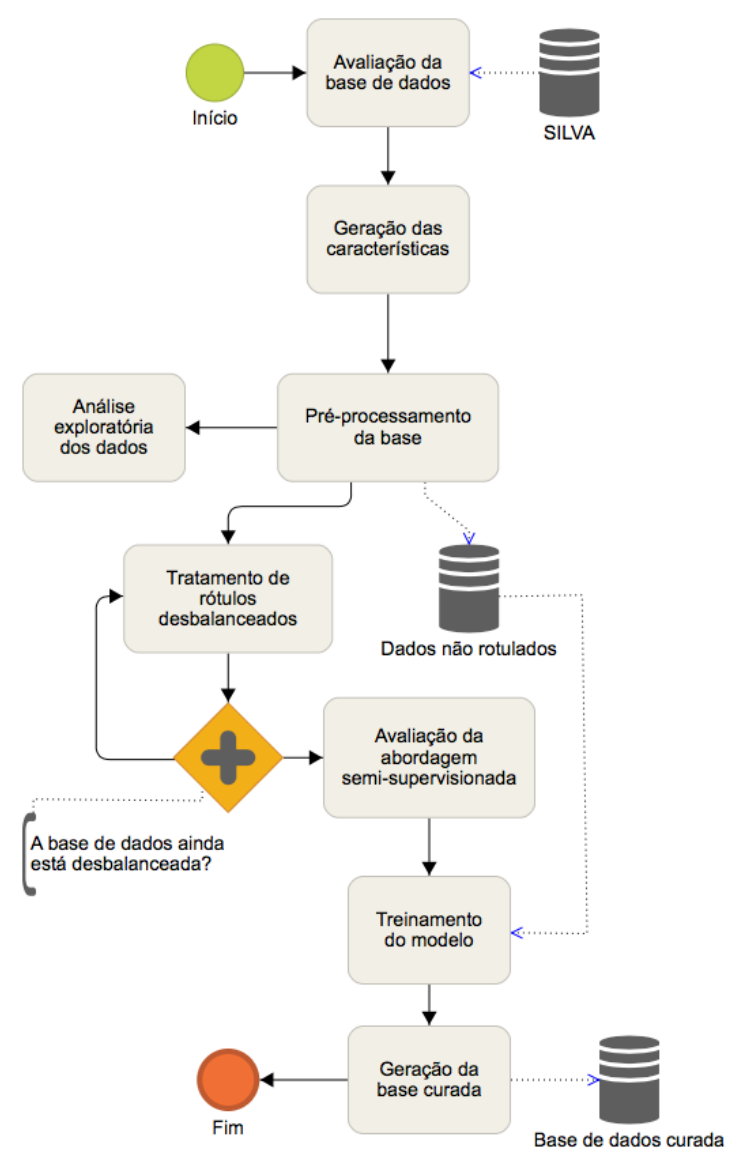

Figura 1. Fluxograma da metodologia utilizada

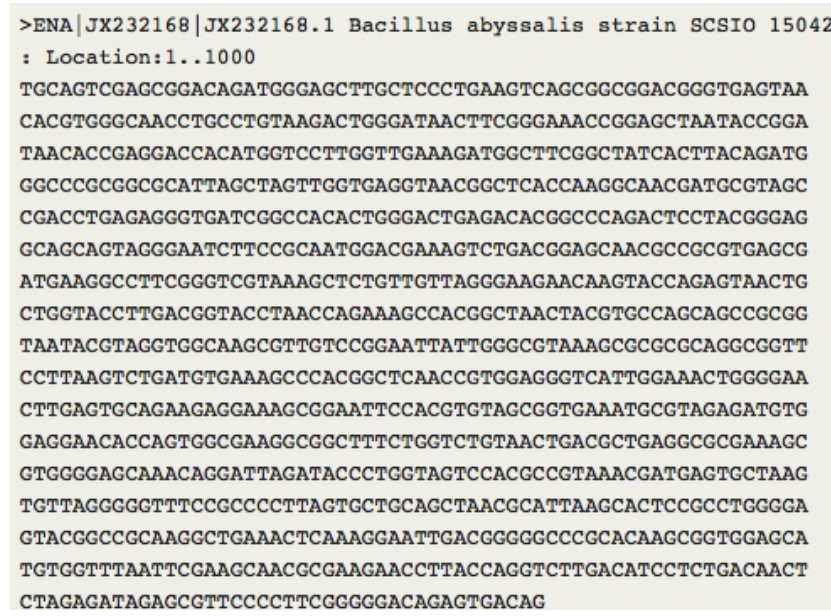

Figura 2. Sequência biológica que caracteriza cada dado do dataset selecionado

de alinhamento (Sedlar et al., 2017). Quando utilizado a rotulagem dependente de alinhamento os algoritmos que as classificam apresentam um problema: são considerados lentos devido à parte que compara as sequências (Sedlar et al., 2017). Já os algoritmos baseados na estratégia independente de alinhamento não possuem essa desvantagem, logo, se tornaram o principal foco de interesse (Sedlar et al., 2017).

A escolha da estratégia para a geração das características e dos parâmetros para cada algoritmo pode afetar significa- 
tivamente o resultado do agrupamento taxonômico. Então, para este projeto foi escolhida a estratégia independente de alinhamento baseada na frequência de tetra nucleotídeos. Através de uma janela deslizante foram contabilizadas a frequência absoluta da combinação quatro a quatro de nucleotídeos presentes na sequência, como por exemplo a frequência de AAAA, AAAT, AAAG, AATA, etc. Assim, ao final do processamento cada item obteve um total de 136 classes.

Após obter a tabela de características de todo o dataset, foi calculado a frequência relativa de cada item, definidos como a razão entre a frequência absoluta e o número total de observações de um item, tornando assim, os itens significativos e comparáveis.

\subsection{Pré-processamento da base de dados}

$\mathrm{Na}$ etapa anterior, foi possível identificar vários dados com rotulagem que não são consideradas espécies, ou seja, sequências sem rótulos que fazem sentido. Dessa forma, sequências com tais rótulos a nível de espécie foram retiradas. Em suma, as classificações retiradas foram: uncultured, unidentified e metagenome.

Também foram retirados dados que possuíam em seu rótulo o final "spp". Tal notação indica que a sequência foi rotulada apenas a nível de gênero. Por fim, foram retiradas também espécies que possuíam menos de 3 sequências no banco. Esse corte foi feito porque não seria possível treinar um modelo no qual um rótulo possuísse apenas duas representações.

Por fim, todos os dados retirados foram classificados como dados não rotulados e serão utilizados para serem reclassificados pelo modelo futuramente.

\subsection{Análise exploratória dos dados}

As sequências do dataset selecionado quando visualizadas detalhadamente apresentaram algumas discordâncias no nível de classificação taxonômica. Como por exemplo, uma sequência classificada a nível de espécie como Bacillus Subtilis, a nível de gênero ela foi classificada erroneamente como Escherichia, no qual o certo seria o gênero Bacillus.

Portanto, através desse problema foi desenvolvido uma análise considerando cada rotulagem como uma classificação e avaliado separadamente cada classe dessa rotulagem. Para cada classe foi feito uma análise gráfica dos dados levando em consideração as diferenças entre os atributos. Essas diferenças calculam a mediana e os interquartis de cada atributo.

Foi gerado então uma matriz de confusão para cada classe, considerando a seguinte avaliação:

- Verdadeiros Positivos (TP): classificados corretamente a nível de espécie e genêro, e que estavam dentro do $1^{\circ}$ e $3^{\circ}$ interquartil gerado;

- Verdadeiros Negativos (TN): classificados incorretamente a nível de espécie e genêro, e que não estavam dentro do $1^{\circ}$ e $3^{\circ}$ interquartil gerado;

- Falsos Positivos (FP): classificados corretamente a nível de espécie e genêro, e que não estavam dentro do $1^{\circ}$ e $3^{\circ}$ interquartil gerado;
Figura 3. Classificação por espécie

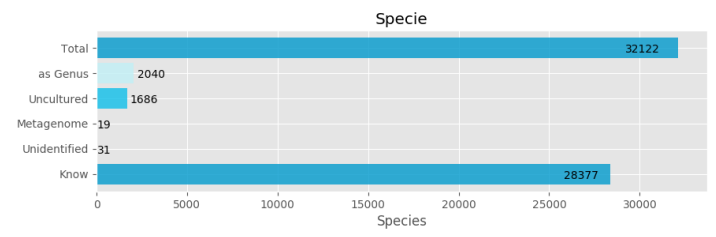

- Falsos Negativos (FN): classificados incorretamente a nível de espécie e genêro, e que estavam dentro do $1^{\circ}$ e $3^{\circ}$ interquartil gerado;

Foram avaliadas as métricas de especificidade (TNR) e sensibilidade (TPR) conforme as fórmulas 1 e 2 , respectivamente.

$$
\begin{gathered}
T N R=T N /(T N+F N) \\
T P R=T P /(T P+F P)
\end{gathered}
$$

\subsection{Avaliação da abordagem semi-supervisionada}

Após a avaliação da base de dados, entendeu-se que a melhor escolha para resolver os problemas identificados seria a utilização de uma abordagem semi-supervisionada . Este paradigma gera modelos na presença de dados rotulados e não rotulados (Triguero et al., 2015). Para avaliações do modelo foi implementado o algoritmo Pseudo-Label (Lee, 2013), o qual segue a estratégia demostrada no Algoritmo 1.

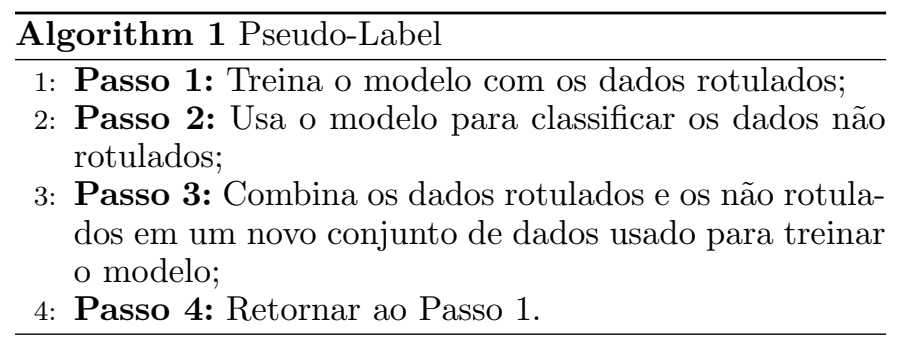

Como métrica de avaliação foi utilizado o coeficiente de determinação, também chamado de $R^{2}$ (R quadrático), uma medida de ajuste de um modelo estatístico linear generalizado que leva em consideração a relação entre os valores observados. O $R^{2}$ varia entre 0 e 1 , indicando, em percentagem, o quanto o modelo consegue explicar os valores observados. Quanto maior o $R^{2}$ mais explicativo é o modelo e melhor ele se ajusta à amostra.

\section{RESULTADOS E DISCUSSÕES}

A princípio, a base de dados SILVA apresentou um total de 32.122 espécies e um total de 1.719.974 sequências, como é possível observar na Figura 3. Entretanto, 63\% das espécies possuem apenas 2 sequências ou menos, não podendo assim, ser utilizada como conjunto de treino. Os outros $9 \%$ são representados por espécies classificadas como uncultured, unidentified, metagenome e com classificação "sp.". Assim, sobraram apenas 28\% das espécies como prováveis espécies a serem utilizadas para treino do modelo.

Em relação a avaliação dos dados caracterizados por sequência, como pode ser observado na Figura 4, é possível verificar que o mesmo não acontece como espécie. $67 \%$ 
Figura 4. Classificação por quantidade de sequências

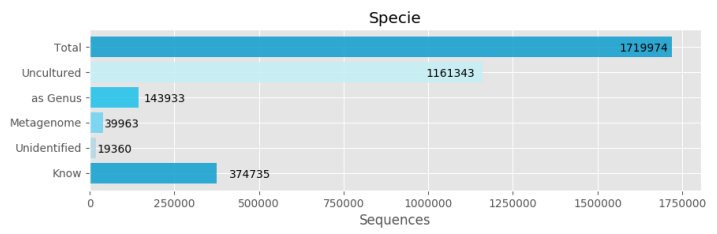

Figura 5. Relação entre a espécie e sua quantidade de sequências

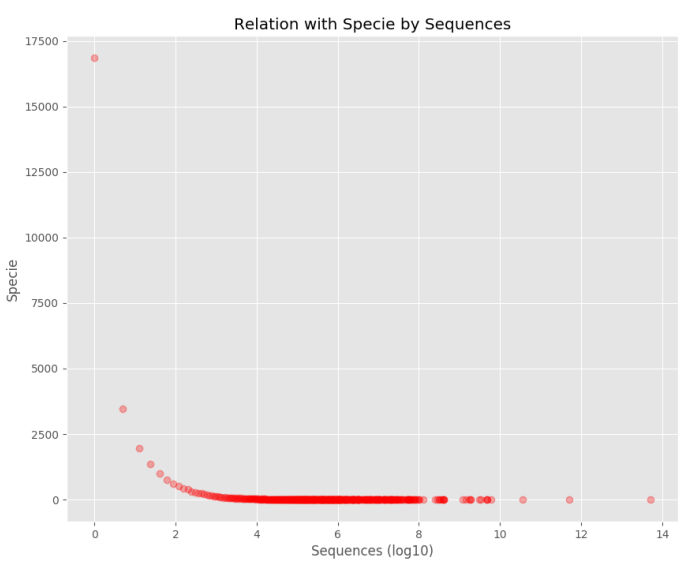

Figura 6. Histograma da quantidade de sequências por espécie

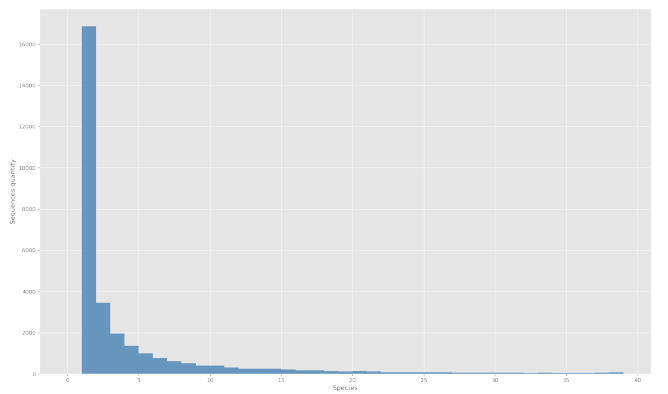

das sequências são consideradas uncultured, ou seja não possuem uma classificação taxonômica.

Fazendo um comparativo entre espécies e a quantidade de sequências que cada uma possui, como pode ser visto na Figura 5, foi possível observar que existe muitas espécies com menos de 3 sequências, ou seja menos de 3 dados estão representando uma rotulagem, e também possui uma única espécie com uma quantidade de dados superior a todas as outras (mais de 800 mil rótulos). Este desbalanceamento entre classes é tão grande que não foi possível demonstrar a grande calda longa da distribuição dos dados em um histograma sem que se fosse necessário separa-lo em sessões (Figura 6).

Na construção da matriz de confusão, cada espécie foi analisada separadamente. Considerando as sequências classificadas como Staphylococcus aureus, a Figura 7 demonstra apenas uma variação de uma característica em relação a especificidade, entretanto, o mesmo não acontece com a
Figura 7. Especificidade e sensibilidade da espécie Staphylococcus aureus

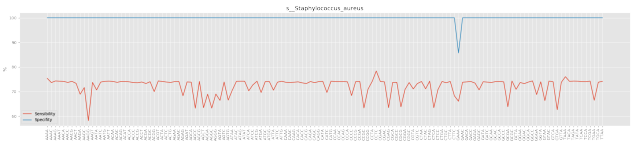

Figura 8. Especificidade e sensibilidade da espécie Escherichia coli

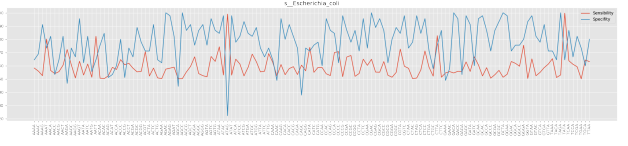

Figura 9. Especificidade e sensibilidade da espécie Acholeplasma palmae

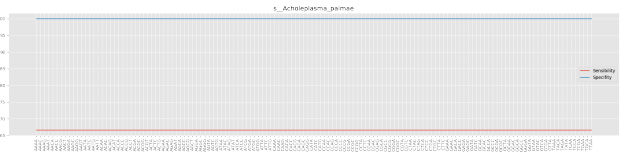

Figura 10. Especificidade e sensibilidade da espécie Acidocella facilis

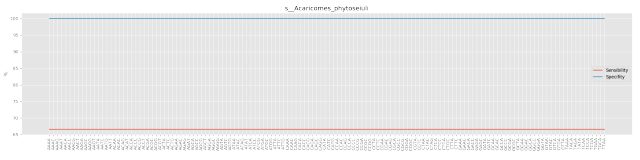

sensibilidade, que varia entre 60 e $80 \%$. Avaliando também a espécie Escherichia coli (Figura 8), o mesmo não acontece, pois apresenta uma alta variação da especificidade e sensibilidade, pode dizer em sua maioria entre 50 e $100 \%$.

Avaliando agora rótulos que possuem poucos dados, como a espécie Acholeplasma palmae e Acidocella facilis, respectivamente representadas pelas Figura 9 e 10, é possível visualizar que tais dados não possuem uma variação de especificidade e sensibilidade comparadas a rótulos que possuem muitos dados.

Logo, através destas análises de rotulagem separadas é possível concluir que, para a modelagem do classificador, deverão ser feitos ajustes de acordo com a rotulagem. Nesse sentido, o ajuste com maior relevância para o objetivo deste trabalho é a sensibilidade, pois é mais importante a capacidade do modelo predizer corretamente, do que fazer a correção de rotulagem erradas.

\section{CONCLUSÕES E TRABALHOS FUTUROS}

Esse trabalho teve como objetivo analisar os problemas de desbalanceamento existentes na base de dados de classificação taxonômica SILVA. Esta é uma base de dados referência em seu domínio, porém, apresenta muitas inconsistências em seus dados, inconsistências estas que podem chegar a invalidar estudos que os utilizem. Sendo assim, em trabalhos futuros, serão utilizados modelos robustos de aprendizado de máquina para corrigir os erros e gerar uma nova base mais confiável e curada.

Com os resultados da análise da base de dados, e na etapa de pre-processamento, foi possível visualizar um grande volume de dados não rotulados, assim uma estratégia de classificação relevante para este problema será a imple- 
mentação de uma abordagem semi-supervisionada. Será utilizado o Aprendizado Ativo, que tem a premissa de selecionar os dados que serão utilizados para a aprendizagem, melhorando o desempenho de métodos tradicionais e problemas com dados desbalanceados.

É possível concluir com tais análises que as etapas de pré-processamento da base de dados têm um alto custo mas, com os resultados demonstrados até o momento, esta fase é de extrema importância para geração de um modelo confiável.

\section{REFERÊNCIAS}

(2006). Greengenes, a chimera-checked 16S rRNA gene database and workbench compatible with ARB. Applied and environmental microbiology, 72(7), 5069-5072. doi: 10.1128/aem.03006-05.

(2014). Ribosomal database project: Data and tools for high throughput rrna analysis. Nucleic Acids Research, 42(D1).

Balvočiūtè, M. and Huson, D.H. (2017). Silva, rdp, greengenes, ncbi and ott - how do these taxonomies compare? BMC Genomics, 18(2), 114.

Beiko, R.G. (2015). Microbial malaise: how can we classify the microbiome? Trends in microbiology, 23(11), 671679 .

Bálint, M., Bahram, M., Eren, A.M., Faust, K., Fuhrman, J.A., Lindahl, B., O'Hara, R.B., Öpik, M., Sogin, M.L., Unterseher, M., and Tedersoo, L. (2016). Millions of reads, thousands of taxa: microbial community structure and associations analyzed via marker genes. FEMS Microbiology Reviews, 40(5), 686-700.

Callahan, B.J., McMurdie, P.J., Rosen, M.J., Han, A.W., Johnson, A.J.A., and Holmes, S.P. (2016). Dada2: high-resolution sample inference from illumina amplicon data. Nature methods, 13(7), 581.

Coordinators, N.R. (2013). Database resources of the national center for biotechnology information. Nucleic Acids Research, 41(D1), D8-D20.

Evtushenko, L., I. Krausova, V., and Yoon, J.H. (2015). Bergey's manual of systematics of archaea and bacteria. $1-81$.

Handelsman, J. (2004). Metagenomics: Application of genomics to uncultured microorganisms. Microbiology and Molecular Biology Reviews, 68(4), 669-685.

Kuczynski, J., Stombaugh, J., Walters, W.A., González, A., Caporaso, J.G., and Knight, R. (2011). Using qiime to analyze $16 \mathrm{~s}$ rrna gene sequences from microbial communities. Current protocols in bioinformatics, 36(1), 10-7.

Kurokawa, K., Itoh, T., Kuwahara, T., Oshima, K., Toh, H., Toyoda, A., Takami, H., Morita, H., Sharma, V.K., Srivastava, T.P., Taylor, T.D., Noguchi, H., Mori, H. Ogura, Y., Ehrlich, D.S., Itoh, K., Takagi, T., Sakaki, Y., Hayashi, T., and Hattori, M. (2007). Comparative metagenomics revealed commonly enriched gene sets in human gut microbiomes. DNA Research, 14(4), 169181.

Lee, D.H. (2013). Pseudo-label : The simple and efficient semi-supervised learning method for deep neural networks.

Nakamura, Y., on behalf of the International Nucleotide Sequence Database Collaboration, Cochrane, G., on behalf of the International Nucleotide Sequence Database Collaboration, Karsch-Mizrachi, I., and on behalf of the International Nucleotide Sequence Database Collaboration (2013). The international nucleotide sequence database collaboration. Nucleic Acids Research, 41(D1), D21-D24. doi:10.1093/nar/gks1084. URL http://dx.doi.org/10.1093/nar/gks1084.

Nguyen, N.P., Warnow, T., Pop, M., and White, B. (2016). A perspective on $16 \mathrm{~s}$ rrna operational taxonomic unit clustering using sequence similarity. NPJ biofilms and microbiomes, 2, 16004.

Parte, A.C. (2014). Lpsn-list of prokaryotic names with standing in nomenclature. In Nucleic Acids Research.

Pires, D.E., Oliveira, F.S., Correa, F.B., Morais, D.K., and Fernandes, G.R. (2018). Tag. me: Taxonomic assignment of genetic markers for ecology. bioRxiv, 263293.

Ravin, N.V., Mardanov, A.V., and Skryabin, K.G. (2015). Metagenomics as a tool for the investigation of uncultured microorganisms. Russian Journal of Genetics, 51(5), 431-439.

Sedlar, K., Kupkova, K., and Provazník, I. (2017). Bioinformatics strategies for taxonomy independent binning and visualization of sequences in shotgun metagenomics. In Computational and structural biotechnology journal.

Settles, B. (2009). Active learning literature survey. Technical report, University of Wisconsin-Madison Department of Computer Sciences.

Teeling, H. and Glöckner, F.O. (2012). Current opportunities and challenges in microbial metagenome analysis - a bioinformatic perspective. Briefings in Bioinformatics, 13(6), 728-742. doi:10.1093/bib/bbs039. URL http:// dx.doi.org/10.1093/bib/bbs039.

Thompson, C.C., Chimetto, L., Edwards, R.A., Swings, J., Stackebrandt, E., and Thompson, F.L. (2013). Microbial genomic taxonomy. BMC Genomics, 14(1), 913.

Triguero, I., García, S., and Herrera, F. (2015). Selflabeled techniques for semi-supervised learning: taxonomy, software and empirical study. Knowledge and Information Systems, 42(2), 245-284. doi:10.1007/ s10115-013-0706-y. URL https://doi.org/10.1007/ s10115-013-0706-y.

Yilmaz, P., Parfrey, L.W., Yarza, P., Gerken, J., Pruesse, E., Quast, C., Schweer, T., Peplies, J., Ludwig, W., and Glöckner, F.O. (2014). The silva and "all-species living tree project (ltp)" taxonomic frameworks. Nucleic Acids Research, 42(D1), D643-D648. 